\title{
Numerical estimate of multi-species ion sound speed of Langmuir probe interpretations in edge plasmas of Wendelstein 7-X
}

\author{
J. COSFELD ${ }^{1}$, P. DREWS ${ }^{1}$, B. BLACKWELL ${ }^{3}$, M. \\ JAKUBOWSKI $^{4}$, H. NIEMANN ${ }^{4}$, D. ZHANG ${ }^{4}$, Y. FENG ${ }^{4}$, \\ and the Wendelstein 7 -X Team ${ }^{1,2,3,4}$ \\ ${ }^{1}$ Forschungszentrum Jülich GmbH, Insitut für Energie- und Klimaforschung - \\ Plasmaphysik, Partner of the Trilateral Euregio Cluster (TEC), 52425 Jülich, \\ Germany \\ 2 JARA-HPC, Jülich Supercomputing Centre, Forschungszentrum Jülich GmbH, \\ 52425 Jülich, Germany \\ ${ }^{3}$ Australian National University, Acton ACT, 2601 Canberra, Australia \\ ${ }^{4}$ Max-Plank-Insitut für Plasmaphysik, 17491 Greifswald / 85748 Garching, Germany \\ E-mail: j.cosfeld@fz-juelich.de
}

\begin{abstract}
The recently modified EMC3-EIRENE code package has been widely applied as an edge-plasma analysis tool and resulted in successful validation against various measured trends seen in stellarator and tokamak plasma boundaries. It was shown that the code package applied for Wendelstein 7-X (W7-X) discharges in interpretive mode can assess the impact of impurity effects on the electron density, measured by a set of Langmuir probes. In particular the spatial quantification of impurities and effects from the effective charge state $Z_{\text {eff }}$ and effective mass $m_{\text {eff }}$, which are non-trivial to record by diagnostics, were examined. The results showed that earlier assumptions of the effective charge-state distribution and effective mass for reported Langmuir probe measurements must be revised. Subsequently, reprocessing these measurements with code-interpreted spatial profiles of the effective charge state and effective mass led to an overall improved physical consistency.
\end{abstract}

Keywords: Wendelstein 7-X, plasma-wall interaction, numerical diagnostics, EMC3EIRENE 


\section{Introduction}

Wendelstein 7-X (W7-X) [1] represents one of the most modern magnetic confinement fusion experiments today. It is based on an optimized magnetic stellarator configuration. W7-X has a modular set of 50 non-planar and 10 planar coils to favorably shape the geometry of the magnetic field [2]. Either the experimental vessel can be twisted realized for the Large Helical Device (LHD) [3] - or external coils can be twisted to achieve the helical twist of the magnetic field lines; the latter is done for W7-X. The magnetic topology of the experiment is a result of its five-fold symmetry. The W7-X has five identical modules with updown symmetry between two halves of each module. However, plasma dynamics within the complex three-dimensional (3D) structure of a stellarator can only partially be assessed using the installed diagnostics.

The simulations presented in this work are consistent with several plasma-edge diagnostics. The deduced informations of the effective charge state and mass are included in a refinement process of the Langmuir probe measurement interpretations. In the present work, a consistent reconstruction of $Z_{\text {eff }}$ for a plasma with carbon as its only impurity is achieved through an iterative process between EMC3-EIRENE simulations and refined Langmuir probe measurement data-sets. This approach includes convergence criteria for deduced plasma parameters, which led to a final reconstructed charge state $Z_{\text {eff }}$ and final effective mass $m_{\text {eff }}$. Through a combination of comparison and reconstruction processes, EMC3-EIRENE is applied as a 3D interpretive tool for W7-X discharges with carbon impurities. A discussion is presented which questions the validity of $Z=1.0$ and $m_{\mathrm{i}}=1.0$. Therefore, the sound speed for a single species plasma had to be replaced by a multi-species one. At first glance, reinterpreting the Langmuir probe measurements presented in this work appears to be irrelevant. However, these Langmuir probe measurements were used as reference points to consistently assess missing $Z_{\text {eff }}$ and $m_{\text {eff }}$ profiles.

In its first operational campaign, W7-X was operated in limiter configuration $[4,5]$. Different Langmuir probe diagnostics were installed on the probe head of a multipurpose manipulator (MPM) [6-8]. The MPM was installed at the low field side of the vacuum vessel [6] at an poloidal angle of $\varphi \approx 200.7^{\circ}$. A plunge of $35 \mathrm{~cm}$ into the vessel was possible for the first operational phase, the last closed flux surface (LCFS) of the plasma was not reachable. However, the interpretation of these Langmuir probe measurements requires information on the impurity content in the plasma, which is thus far missing. Previous studies for impurity effects in an experimental fusion plasma $[9,10]$ showed that these must not be neglected in studies of the plasma edge. The exact quantification of the impurity content in the plasma is provided by every single chargestate density of the impurity species, assessed by EMC3-EIRENE simulations. These are included in the iterative processes presented in this work. However, the effective chargestate distribution $Z_{\text {eff }}$ and effective mass $m_{\text {eff }}$ are utilized as a visual quantification of the impurity content in the plasma [11] rather than every single charge-state density because they are more legible. The effective charge state and mass are calculated from 
each charge-state density $n_{i}$, where $i$ gives the charge state number,

$$
Z_{\mathrm{eff}}=\frac{\sum_{i} n_{i} Z_{i}^{2}}{\sum_{i} n_{i} Z_{i}} \quad, \quad m_{\mathrm{eff}}=\frac{\sum_{i} n_{i} m_{i}}{\sum_{i} n_{i}} .
$$

An overview of the each charge state density is given in the supplement at two different cross-sections. The impurity ion mass is given by $m_{i}$ and the atomic charge number is $Z_{i}$. Effective charge state and mass measurements were not available for the limiter configuration of W7-X. Thus, a constant value or profile is commonly assumed for $Z_{\text {eff }}$ and $m_{\text {eff }}$ in the Langmuir probe data interpretations which were questioned in this work. The remainder of this work is organized as follows. Section 2 provides an overview of the conducted EMC3-EIRENE simulations, section 3 investigates the beneficial impact of the EMC3-EIRENE simulations on Langmuir probe measurements, resulting in an assessment of internal consistency between different Langmuir probe data-sets, section 4 describes and discusses the iterative process used to reconstruct the effective charge state and effective mass, and finally, section 5 summarizes the main results and concludes the present work.

\section{EMC3-EIRENE impurity simulations}

For this work, the five-fold periodicity and the up-down stellarator symmetry [1] were taken into account. Therefore, the EMC3-EIRENE simulation grid in this work covered a toroidal segment of $\Delta \varphi_{\text {tor }}=36.0^{\circ}=72 \delta \varphi_{\text {tor }}$ (where $\delta \varphi_{\text {tor }}$ denotes the toroidal discretization) with a resolution of $\Delta R=0.44 \mathrm{~cm}$ in a radial direction, with a poloidal grid for each of the 72 poloidal cross-sections (with a poloidal discretization of $\delta \theta_{\mathrm{pol}}=0.7^{\circ}$ ). EMC3-EIRENE simulations were conducted on this grid to assess the plasma edge dynamics with a full $3 \mathrm{D}$ resolution. The free simulation parameters are the diffusion coefficient, which depends on the thermal conductivity of the electrons and ions; $D_{\perp}=3 \chi_{\mathrm{e}, \perp}=3 \chi_{\mathrm{i}, \perp}\left(\chi_{\mathrm{e}, \perp}\right.$ and $\chi_{\mathrm{i}, \perp}$ are grouped to $\chi_{\perp}$ because they are held the same throughout this work); the input heating power entering the scrape-off layer (SOL) $P_{\text {heat }}^{\mathrm{SOL}}$ t the power radiated in the SOL $P_{\mathrm{rad}}^{\mathrm{SOL}}$; and the electron density $n_{\mathrm{e}}$ at the separatrix. Previous comparison studies [12] between EMC3-EIRENE simulations and plasma edge diagnostic findings show that for a given magnetic topology - and its connection length (named $L_{\mathrm{c}}$ ) pattern - non-uniform cross-field transport coefficients need to be considered. Values for $D_{\perp}=3 \chi_{\perp}$ are then replaced with spatial profiles, as it is shown in figure 5 from [12]. Strong variations in the connection length pattern of the Large Helical Device (LHD) demand an applied Heaviside step function, for the non-uniform cross-field transport coefficients, with $\chi_{\perp}=0.2 \mathrm{~m}^{2} / \mathrm{s}$ for $L_{\mathrm{c}} \gtrsim 10^{3} \mathrm{~m}$ and $\chi_{\perp}=4.0 \mathrm{~m}^{2} / \mathrm{s}$ for $L_{\mathrm{c}} \lesssim 10^{3} \mathrm{~m}$. However, changes between different $L_{\mathrm{c}}$ regions of $\mathrm{W} 7-\mathrm{X}$ 's magnetic topology are smaller - rather steps between 20 and $80 \mathrm{~m}$ exist (see figure 3.3 from [13]) - than a step from $10^{2} \mathrm{~m}$ to $10^{4} \mathrm{~m}$. Thus, it is reasonable to neglect changes to $D_{\perp}$ and $\chi_{\perp}$ for the presented simulations in this work.

Free parameters were chosen to match the plasma scenario described in figure 1, which 
involves the aforementioned limiter Langmuir probe measurements (figures 1(c) and (d)). The bolometric measurement of $P_{\text {rad }}$ provides a measure of the power that is radiated in total $P_{\mathrm{rad}}^{\mathrm{total}}$, of which a fraction is radiated in the scrape-off layer $P_{\mathrm{rad}}^{\mathrm{SOL}}$. The simulation requires the heating power to enter the SOL as an input parameter. Therefore, power that is lost in the SOL must be subtracted from the overall lost power to account for the power loss in the core region (not simulated here). Considered discharges were selected because MPM Langmuir probe measurements are only available for a small subset of discharges. The MPM plunged into the plasma over a time interval of $\Delta t \approx 0.057 \mathrm{~s}$ during the discharge time (see the dashed black lines in figure 1). The resulting plasma parameters (measured by MPM Langmuir probes, see figures 1(c) and (d)) during the plunge time interval were used as input parameters for the EMC3-EIRENE simulations. An overall heating power of 2.00 MW was applied, of which a total of $0.80 \mathrm{MW}$ was radiated, and thus lost. Considering all possible power losses, this was summed to a heating power entering the $\mathrm{SOL}$ of $P_{\text {heat }}^{\mathrm{SOL}}=1.32 \mathrm{MW}$ and a radiated power in the SOL of $P_{\mathrm{rad}}^{\mathrm{SOL}}=120 \mathrm{~kW}$. One obtains the input density and temperature by an extrapolation from the deepest point of the MPM plunge path to the LCFS. The electron density and temperature were constrained to $n_{\mathrm{e}}^{\mathrm{LCFS}}=3.000 \times 10^{18} \mathrm{~m}^{-3}$ and $T_{\mathrm{e}}^{\mathrm{LCFS}}=25 \mathrm{eV}$. From here on, $\Lambda$ refers to this simulation setup. The radial spatial resolution of the MPM allowed for a detailled comparison between simulations and measurements [6]. The plasma-edge profiles from the EMC3-EIRENE simulations were compared with the experimental data-sets, which resulted in a general overall agreement and consistency at the MPM Langmuir probe measurement location (see figure 2), because these were used as input parameters for simulation $\Lambda$. Thus this consistency between plasma edge profiles $\left(T_{\mathrm{e}}\right.$ and $\left.n_{\mathrm{e}}\right)$ assessed by EMC3-EIRENE simulations and MPM Langmuir probe measurements is enforced. But, a small offset between $n_{\mathrm{e}, \mathrm{MPM}}$ and $n_{\mathrm{e}, \mathrm{EMC} 3}$ remained for the radial-most outer measurement points of the MPM (see figure 2). However, this small offset was neglected in respect of the overall consistency. Simulation $\Lambda$ did not only shown a consistency to MPM measurement shown in figure 2, but to limiter Langmuir probes and heat-flux measurements onto the limiter [14]. Though, a detailed discussion of these measurements is beyond of the scope of this work. Hence wise, the refinement of MPM Langmuir probes using EMC3-EIRENE code-outputs is the focus in the following. 

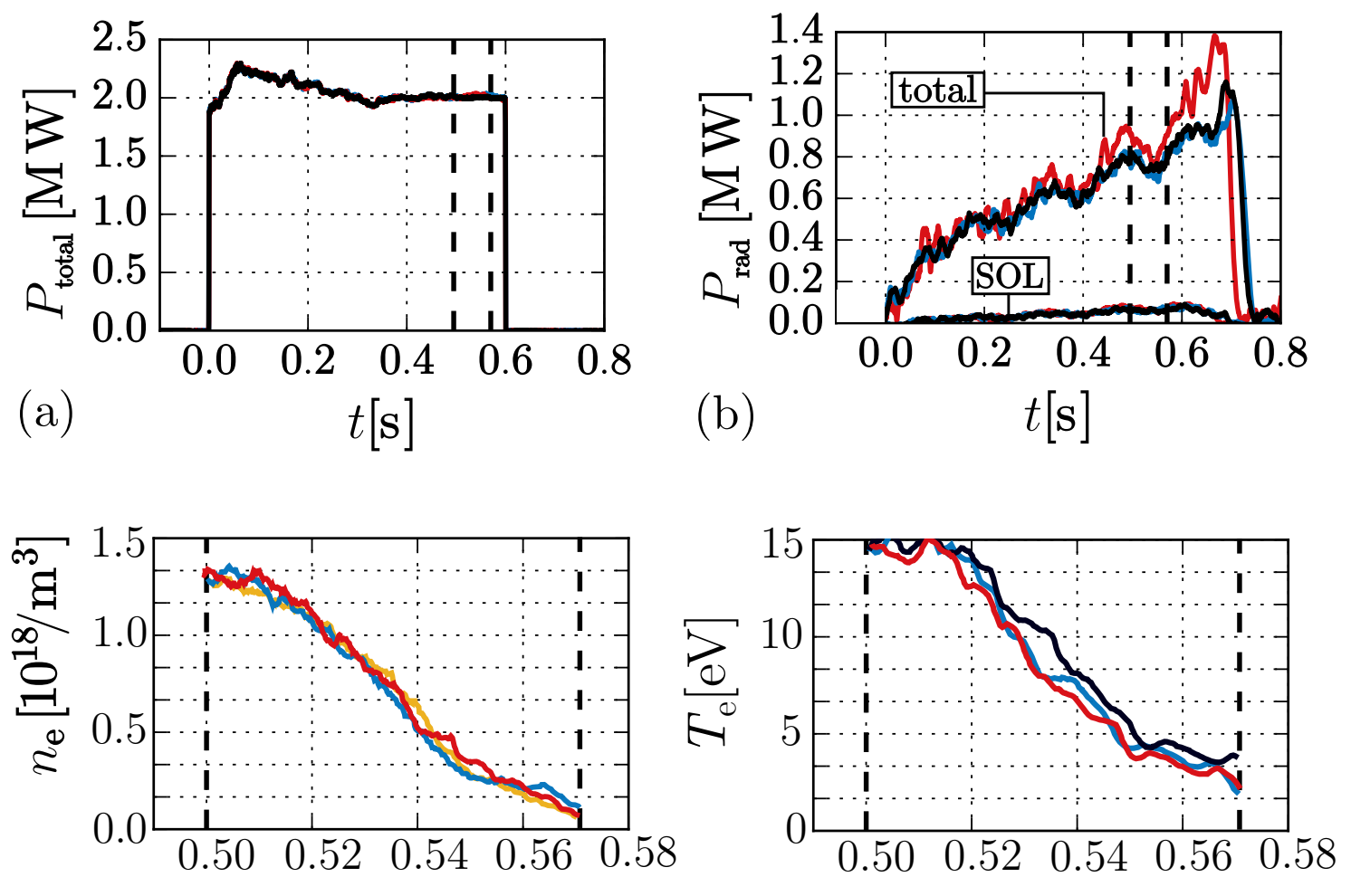

(c)

$t[\mathbf{s}]$

(d)

$t[\mathbf{s}]$

Figure 1. Overview of guiding measurements of heating power (a), radiated power (b), and $n_{\mathrm{e}}(\mathrm{c})$ and $T_{\mathrm{e}}(\mathrm{d})$ by limiter Langmuir probes. The color code refers to different discharges: black $=$ discharge 20160308.22, red $=$ discharge 20160308.23 and blue $=$ discharge 20160308.24. MPM Langmuir probe measurements were performed at a specific time interval $\Delta t$, indicated by vertical dashed lines.
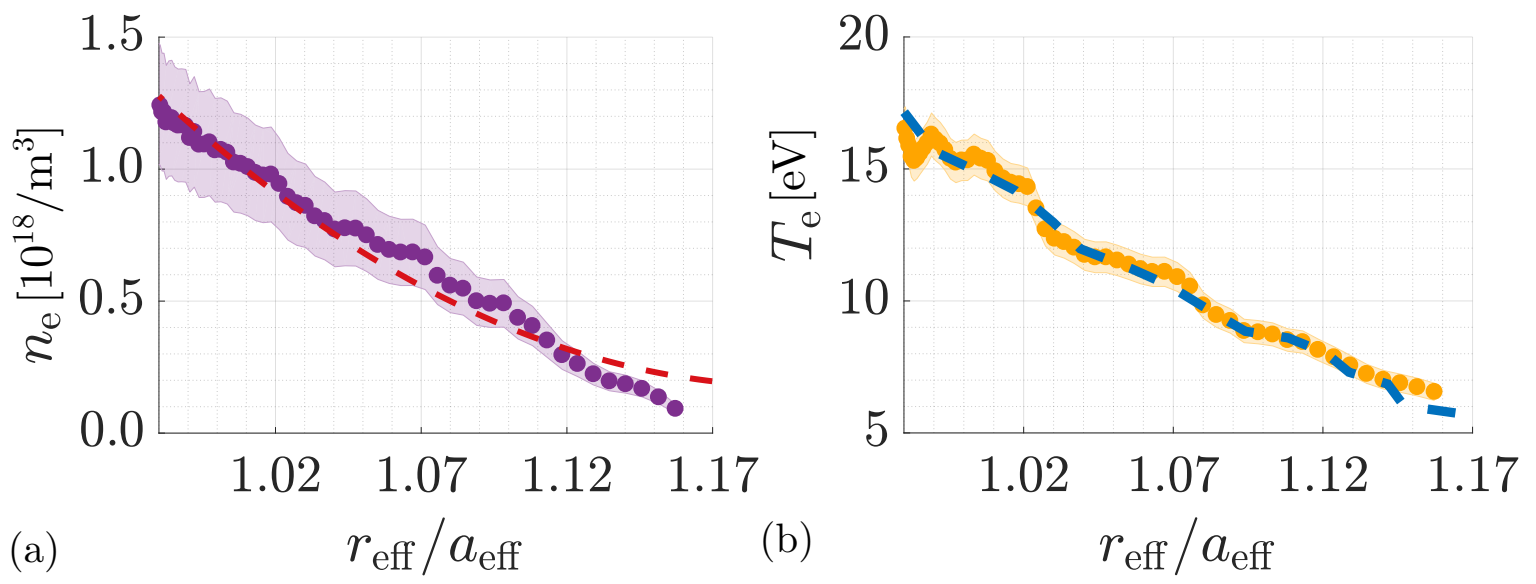

Figure 2. Comparison of density $n_{\mathrm{e}}$ (a) and temperature $T_{\mathrm{e}}$ (b) of EMC3-EIRENE simulation (dashed) and manipulator Langmuir probe measurements (dots, uncertainties of the measurements shown in the shaded region) plotted over the effective radius $r_{\text {eff }}$. Simulation parameters are taken from $\Lambda$. 


\section{3D spatially resolved reconstruction of the effective charge state and effective mass}

In this section, we discuss the reinterpretation of the utilized Langmuir probe measurements, including the assessed charge-state densities from the EMC3-EIRENE simulation $\Lambda$. As previously mentioned, the effective charge state $Z_{\text {eff }}$ and mass $m_{\text {eff }}$ are used to provide readers with easy access to the quantification of the impurity content in the plasma.

Each considered Langmuir probe data-set is updated with its charge-state density profiles from the simulation with which the $n_{\mathrm{e}, \mathrm{MPM}}$ profile was compared before. Hence, a $n_{\text {e,EMC3 }}$ profile that coincides with the profile of the Langmuir probe measurement $n_{\mathrm{e}, \mathrm{MPM}}$ also leads to a convergence of every single charge-state density, and therefore also for the $Z_{\text {eff }}$ and $m_{\text {eff }}$ profiles (see figure 3). An iterative process was applied to the comparison process of $n_{\mathrm{e}, \mathrm{MPM}}$ and $n_{\mathrm{e} \text {,EMC3 }}$, starting with assessed $Z_{\text {eff }}$ and $m_{\text {eff }}$ profiles. These are then used to calculate refined $n_{\mathrm{e}, \mathrm{MPM}}$ which needs to be rematched by $n_{\mathrm{e}, \mathrm{EMC} 3}-$ input parameters need to be tuned - to complete a first iterative step $l$. MPM Langmuir probe measurements were not able to separate the electron temperature $T_{\mathrm{e}}$ from the ion temperature $T_{\mathrm{i}}$ of the plasma. Hence, $T_{\mathrm{e}}$ and $T_{\mathrm{i}}$ were taken from EMC3-EIRENE for this work.

The calculation of the electron density from the saturation current $I_{\text {sat }}$ (used in the data interpretation process in [6]) measured by the Langmuir probes is affected by a change of the ion speed of sound $c_{\mathrm{s}}$,

$$
n_{\mathrm{e}, \mathrm{MPM}}=\frac{I_{\mathrm{sat}}}{0.49 A_{\mathrm{eff}} c_{\mathrm{s}}}
$$

where $A_{\text {eff }}$ is the effective area of the Langmuir pins. Stangeby [15] defines the speed of sound $c_{\mathrm{s}}$ of a single species plasma scenario as,

$$
c_{\mathrm{s}}^{\text {single }}=\sqrt{\frac{T_{\mathrm{i}}+Z T_{\mathrm{e}}}{m_{\mathrm{i}}}},
$$

where the ion mass is denoted with $m_{\mathrm{i}}$, the electron temperature $T_{\mathrm{e}}$, and the ion temperature $T_{\mathrm{i}}$. Stangeby assumes the plasma flow to be isothermal. The adiabatic index $\gamma=1$ was already applied to equation (3). Plasma temperatures are given in $\mathrm{eV}$, and hence the Boltzmann constant $k$ is not introduced in equation (3). Equation (3) is often used to describe plasma scenarios that have carbon or oxygen as second plasma species in addition to hydrogen. Therefore, $Z=1.0$ and $m_{\mathrm{i}}=1.0$ are assumed. The measurement data-sets presented in figure 2 rely on these assumptions. The following discussion questions the validity of $Z=1.0$ and $m_{\mathrm{i}}=1.0$, but first, the sound speed for a single species plasma must be replaced by a multi-species one. An expression for the overall plasma speed of sound $c_{\mathrm{s}}$ for a multi-species plasma was determined by 


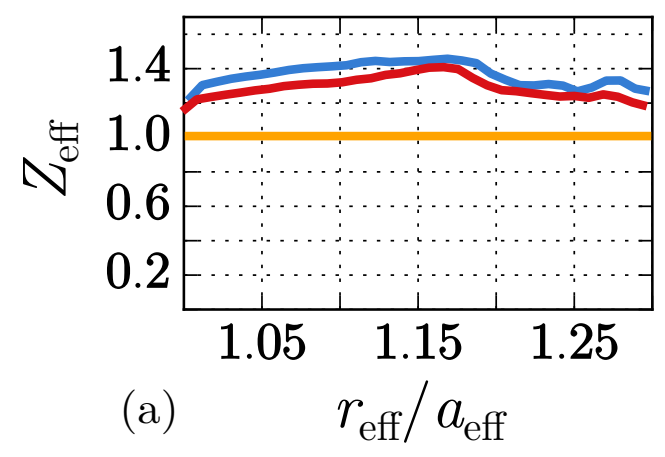

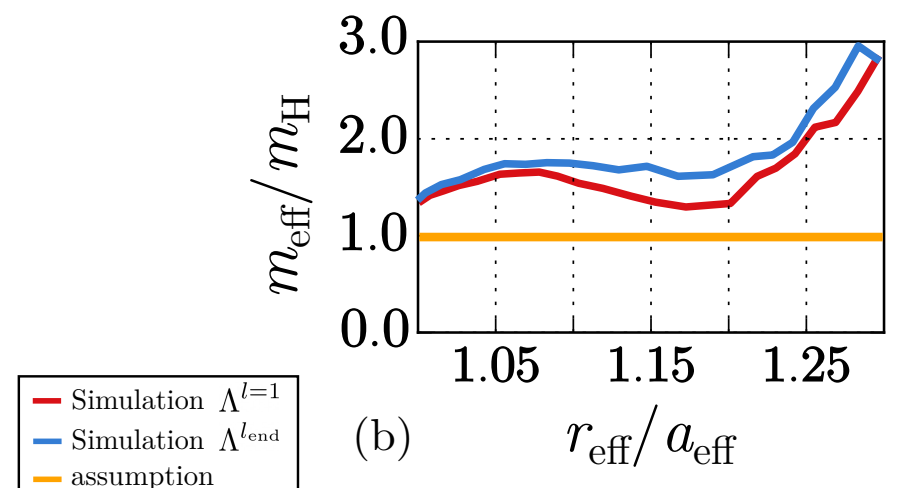

Figure 3. Comparison of the simulated $Z_{\text {eff }}$ (a) and $m_{\text {eff }}$ (b) for simulation $\Lambda^{l=1}$ (red) and $\Lambda^{l_{\text {end }}}$ (blue) taken at the MPM measurement location; plotted with the previously made assumptions of $Z_{\text {eff }}=1$ and $m_{\text {eff }}=1$ (yellow). All results are plotted against the effective radius $r_{\text {eff }}$ normalized to $a_{\text {eff }}$.

Tokar [16],

$$
\begin{aligned}
c_{\mathrm{s}}^{\mathrm{multi}} & =\sqrt{\frac{\sum_{i=0}^{\kappa} \Gamma_{i}\left(T_{i}+Z_{i} T_{\mathrm{e}}\right)}{\sum_{i=0}^{\kappa} \Gamma_{i} m_{i}}}, \quad \Gamma_{i}=n_{i} u_{i}, \\
& =\sqrt{\frac{\sum_{i=0}^{\kappa} n_{i} u_{i}\left(T_{i}+Z_{i} T_{\mathrm{e}}\right)}{\sum_{i=0}^{\kappa} n_{i} u_{i} m_{i}}} .
\end{aligned}
$$

The adiabatic index is again set to $\gamma=1$ and $k$ drops out because of the previously selected units for the plasma temperatures. Furthermore, $u_{i}$ represents the velocity of one plasma species out of $\kappa$ plasma species. Hence, $u_{i}$ is a contribution to the specific plasma species flow $\Gamma_{i}$. Next, $c_{\mathrm{s}}^{\text {multi }}$ calculated from the simulation results of $\Lambda$ can be inserted into equation 2 and yields an electron density that includes the electrons coming from the impurity charge states. However, this is only necessary when the assumptions $\left(Z=Z_{\text {eff }}=1.0\right.$ and $\left.m_{i}=m_{\text {eff }}=1.0\right)$ are no longer valid. This is clearly given for simulation $\Lambda$ because $Z_{\text {eff }}$ and $m_{\text {eff }}$ are no longer equal to 1 , as shown in figure 3. Hence, a recalibration of the measurements (see figure 4) can be conducted using the presumably more plausible charge-state densities (represented in $Z_{\text {eff }}$ and $m_{\text {eff }}$ in this work) from carbon. Obviously, this must be followed by adjusting the simulation parameters, because at least one of the parameter constraints changed visually by the offset between $n_{\mathrm{e}, \mathrm{EMC} 3}$ and $n_{\mathrm{e}, \mathrm{MPM}}$ (see figure 4 ). The constraining discussed in section 2, which was to match measurements with EMC3-ERIENE simulations, must be redone. For this reason, an iterative process is used from this set of EMC3-EIRENE simulations, where each simulation refers to an iterative step $l$. The simulation setups now reach from $\Lambda=\Lambda^{l=1}$ to $\Lambda^{l_{\text {end }}}$. The $Z_{\text {eff }}$ and $m_{\text {eff }}$ profiles are deduced from the simulations in each iterative step $l$ and used to recalibrate the measurement. A schematic overview of the first iterative step is shown in figure 4, and each subfigure (from (a) to (d)) corresponds to the substeps of the first iterative step. This overview discusses the iterative process 


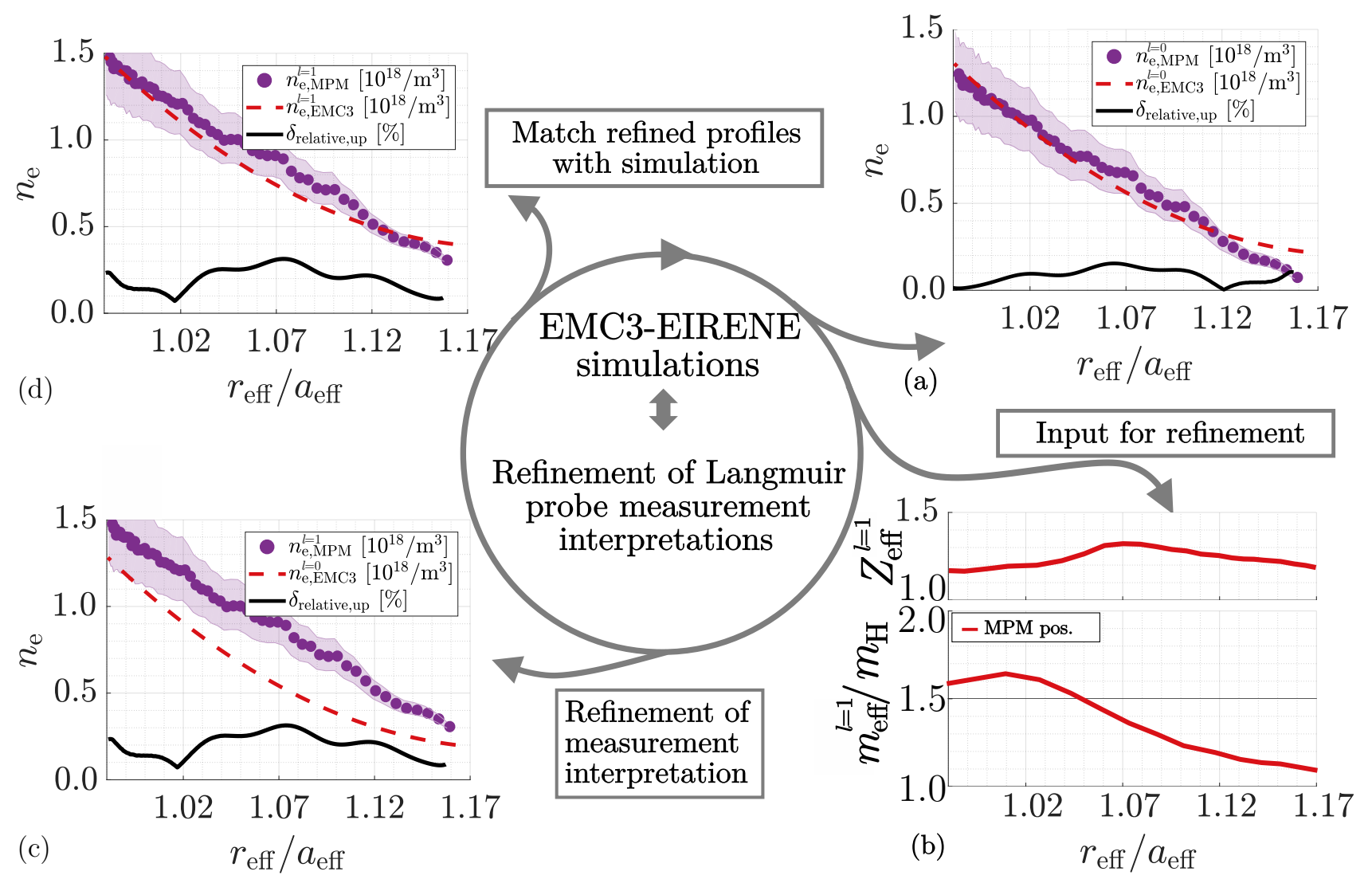

Figure 4. Schematic overview of the first step $l=1$ of the iterative process. Electron density from $\Lambda$ (a), $Z_{\text {eff }}=1.0$ and $m_{\text {eff }}=1.0$ were assumed for $n_{\mathrm{e}, \mathrm{MPM}}$. Deduced $Z_{\text {eff }}^{l}$

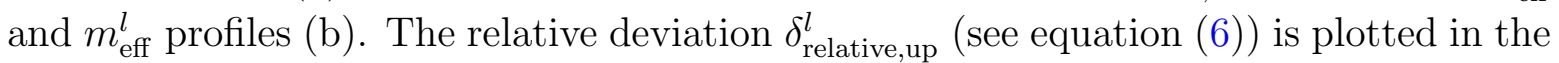
comparison plot of the numeric and measured density data-set (c) and (d). A reworking of figure 2 is shown in $(d)$.

for the MPM measurements. Density profiles measured using the MPM are used as a reference in figure 4 . The first iterative step begins with the assessment of $Z_{\text {eff }}^{l=1}$ and $m_{\text {eff }}^{l=1}$ (substeps (a) and (b), see figure 4) from the $n_{\mathrm{e}}$ profiles shown in figure 2 . In the next substep (c) of figure 4 , an updated $n_{\mathrm{e}, \mathrm{MPM}}^{\Lambda^{l=1}}$ profile including the assessed $Z_{\text {eff }}^{l}$ and $m_{\text {eff }}^{l}$ profile is shown. Hence, one can observe displacement regarding $n_{\mathrm{e}, \mathrm{MPM}}^{l=1}$ through comparing substeps (a) and (c) shown in figure 4. This introduced displacement of $n_{\mathrm{e}, \mathrm{MPM}}^{l=1}$ must be matched with the input parameters of EMC3-EIRENE, and then the input density is changed. For substep (d), a switch from an input density (discussed in section 2) of $n_{\mathrm{e}}=3.000 \times 10^{18} \mathrm{~m}^{-3}$ to $3.126 \times 10^{18} \mathrm{~m}^{-3}$ is applied to match $n_{\mathrm{e}, \mathrm{MPM}}^{l=1}$ of substep (c) with $n_{\mathrm{e}, \mathrm{EMC} 3}^{l=1}$. The first iterative step is completed because consistency between $n_{\mathrm{e}, \mathrm{MPM}}^{l=1}$ and $n_{\mathrm{e}, \mathrm{EMC} 3}^{l=1}$ is achieved again. The relative deviation of the EMC3EIRENE density profiles $n_{\mathrm{e}, \mathrm{EMC} 3}^{l}$ and $n_{\mathrm{e}, \mathrm{MPM}}^{l}$ in the first iterative step is calculated 


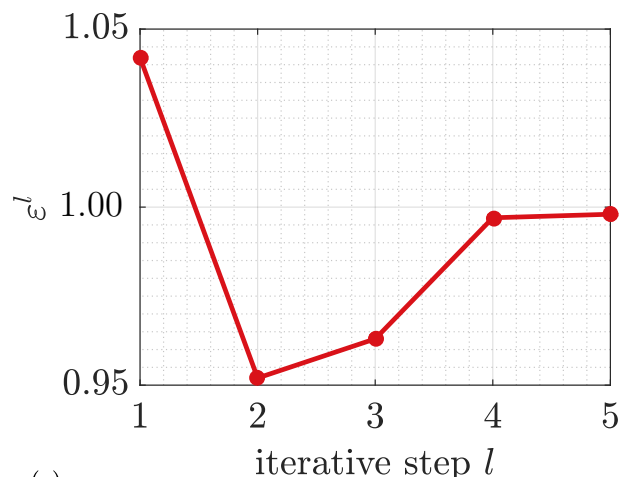

(a)

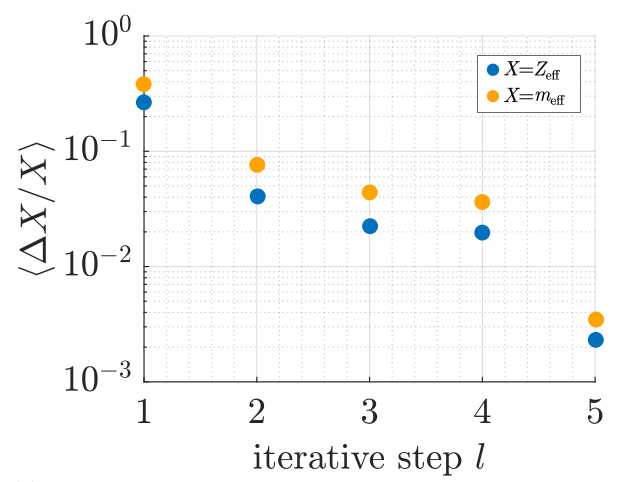

(c)

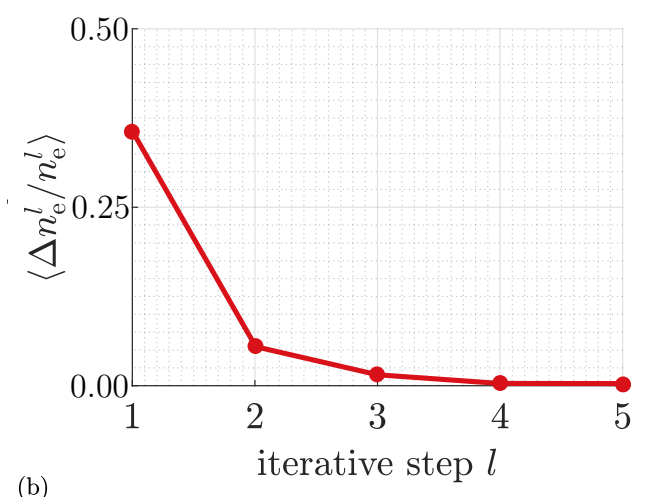

\begin{tabular}{|c|c|c|c|c|c|}
\hline $\begin{array}{c}\text { step } \\
l\end{array}$ & $\begin{array}{c}\text { input } \\
\text { density } \\
{\left[\mathrm{m}^{3} / 10^{18}\right]}\end{array}$ & $\varepsilon^{l}$ & $\begin{array}{r}\left\langle\Delta Z_{\text {eff }}\right. \\
\quad\left|Z_{\text {eff }}\right\rangle\end{array}$ & $\begin{array}{r}\left\langle\Delta m_{\text {eff }}\right. \\
\left.\quad / m_{\text {eff }}\right\rangle\end{array}$ & $\begin{array}{r}\left\langle\Delta n_{\mathrm{e}, \mathrm{MPM}}^{l}\right. \\
\left.\quad / n_{\mathrm{e}, \mathrm{MPM}}^{l}\right\rangle\end{array}$ \\
\hline 1 & 3.000 & 1.042 & 0.2302 & 0.3849 & 0.2674 \\
\hline 2 & 3.1260 & 0.952 & 0.0413 & 0.0762 & 0.0413 \\
\hline 3 & 2.9786 & 0.963 & 0.0221 & 0.0439 & 0.0121 \\
\hline 4 & 2.8702 & 0.997 & 0.0199 & 0.0357 & 0.0032 \\
\hline 5 & 2.8432 & 0.998 & 0.0023 & 0.0035 & 0.003 \\
\hline
\end{tabular}

(d)

Figure 5. Factor $\varepsilon^{l}$ plotted against each iterative step (a). The relative change of the refined MPM measurement of $n_{\mathrm{e}, \mathrm{MPM}}^{l}$ is plotted against each iterative step (b). The relative change of the $Z_{\text {eff }}$ and $m_{\text {eff }}$ profiles is plotted against each iterative step in (c). Simulation parameters are taken from plasma scenario $\Lambda$, but $n_{\mathrm{e}}$ was iterated from $3.000 \times 10^{18} \mathrm{~m}^{-3}$ down to $2.8432 \times 10^{18} \mathrm{~m}^{-3}$. Chart $(\mathrm{d})$ shows the exact values of each iterative step.

using,

$$
\delta_{\text {relative,up }}^{l=1}=\operatorname{mean}\left(\frac{n_{\mathrm{e}, \mathrm{EMC} 3}^{l=1}-n_{\mathrm{e}, \mathrm{MPM}}^{l=1}}{n_{\mathrm{e}, \mathrm{EMC} 3}^{l=1}}\right) .
$$

This measure is used to quantify the convergence of the density profiles to each other. Subsequently, the next iterative steps are conducted following the same scheme of substeps shown and discussed in figure 4. The appropriate factor $\varepsilon^{l}$ for a recalibration of the input density $n_{\mathrm{e}}$ of the simulation for the iterative step $l+1$ can be quantified using (with equations (2) and (5)),

$$
\varepsilon^{l}=\frac{n_{\mathrm{e}, \mathrm{MPM}}^{l}}{n_{\mathrm{e}, \mathrm{MPM}}^{l-1}} .
$$

From here on, $\Lambda^{l_{\text {end }}}$ refers to the simulation setup with the factor $\varepsilon^{l}$ closest to 1 , which refers to the smallest change at the end of the iterative process. Figure 5 (a) shows the relative change of $\varepsilon^{l}$ calculated by equation (7). The relative change for $n_{\mathrm{e}, \mathrm{MPM}}$ (shown 

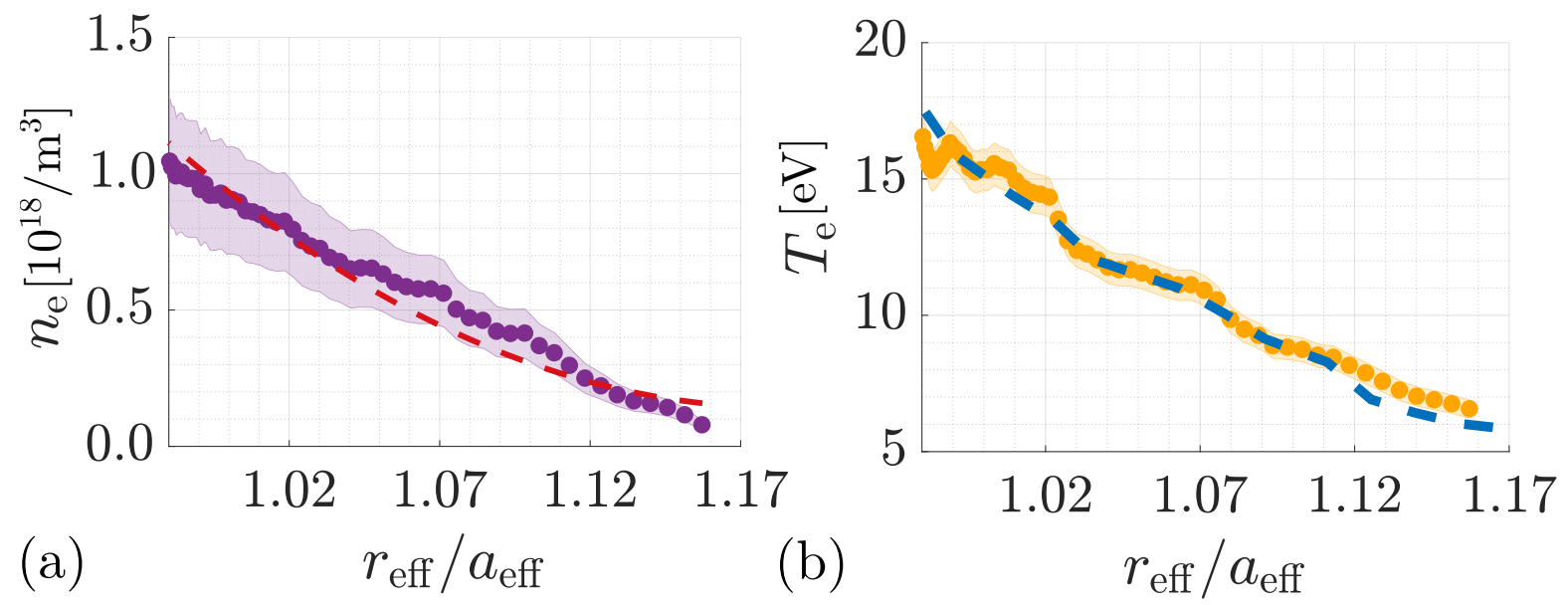

Figure 6. Final comparison between the EMC3-EIRENE simulation (dashed) and $Z_{\text {eff }}^{l_{\text {end }}}$ adjusted manipulator Langmuir probe measurements (dots). Plot (a) shows $n_{\mathrm{e}}$, and (b) shows $T_{\mathrm{e}}$. The simulation parameters used are from plasma scenario $\Lambda^{l_{\text {end }}}$.

in (b) of figure 5), $Z_{\text {eff }}$ and $m_{\text {eff }}$ (both shown in (c) of figure 5 ) drops below $1 \%$ for an input density of $2.8432 \times 10^{18} \mathrm{~m}^{-3}$ (used in the last iterative step). Figure 3 shows $Z_{\text {eff }}$ and $m_{\text {eff }}$ profiles of the first iterative step using the simulation $\Lambda^{l=1}$ and $\Lambda^{l_{\text {end }}}$ at MPM measurement positions. Because the relative deviation of $Z_{\text {eff }}$ and $m_{\text {eff }}$ for the MPM region converges to a value below $1 \%$, an overall agreement between the measurement and simulation is shown as the final result in figure 6 .

Additionally, each simulation setup shown in figure 6 was accompanied by a scan over the cross-field transport coefficients and the radiated Power $P_{\text {rad }}^{\mathrm{SOL}}$. A sensitivity study for $n_{\mathrm{e}, \mathrm{EMC} 3}$ and $T_{\mathrm{e}, \mathrm{EMC} 3}$ concerning the cross-field transport coefficients $D_{\perp}$ and $\chi_{\perp}$, and the radiated power $P_{\text {rad }}^{\mathrm{SOL}}$ was performed to determine the influence of these free simulation parameters. The diffusion coefficient $D_{\perp}$ was chosen in $D_{\perp} \in[0.4,0.5,0.6] \mathrm{m}^{2} / \mathrm{s}, \chi_{\perp}$ is again calculated by $D_{\perp}=3 \chi_{\perp}$. $P_{\mathrm{rad}}^{\mathrm{SOL}}$ was chosen in $P_{\mathrm{rad}}^{\mathrm{SOL}}=[60,120,180] \mathrm{kW}$ for each iterative step. The variation of $D_{\perp}, \chi_{\perp}$, and $P_{\text {rad }}^{\mathrm{SOL}}$ resulted in both cases for each iterative step in profiles, which again showed consistency between $n_{\mathrm{e}, \mathrm{EMC} 3}$ and $n_{\mathrm{e}, \mathrm{MPM}}$. Findings from M. Kobayashi [12] about the small influence of the cross field diffusion on the electron density and temperature, are confirmed by figure 7 . In figure 7 , the resulting change of $n_{\mathrm{e}, \mathrm{EMC} 3}$ is observable in the enclosed areas of $n_{\mathrm{e}, \mathrm{EMC} 3}^{D_{\perp}}, n_{\mathrm{e}, \mathrm{EMC} 3}^{\chi_{\perp}}$, and $n_{\mathrm{e}, \mathrm{EMC} 3}^{P_{\mathrm{rad}}}$. The overlap of the simulated $n_{\mathrm{e}, \mathrm{EMC} 3}$ and measured $n_{\mathrm{e}, \mathrm{MPM}}$ (with error bars) remains high; thus, the consistency between $n_{\mathrm{e}, \mathrm{EMC} 3}$ and $n_{\mathrm{e}, \mathrm{MPM}}$ is preserved concerning the variations of $D_{\perp}$ and $P_{\mathrm{rad}}^{\mathrm{SOL}}$.

In section 2 , simulation $\Lambda$ provided an overall agreement with the MPM Langmuir probe measurements. Here, the simulation $\Lambda^{l_{\text {end }}}$ again shows an overall agreement for the MPM measurements, including a more reasonable estimate for the $Z_{\text {eff }}$ and $m_{\text {eff }}$ profiles. Thus, the resulting final profiles of the MPM measurements (see figure 6) lie at the bottom edge of the uncertainties (refinement of $12 \%$ ) of the unrefined $n_{\mathrm{e}}$ profiles shown in figure 2 (matched by EMC3-EIRENE using $\Lambda$ ). The initial assumption of $Z_{\text {eff }}$ and $m_{\text {eff }}$ to be 


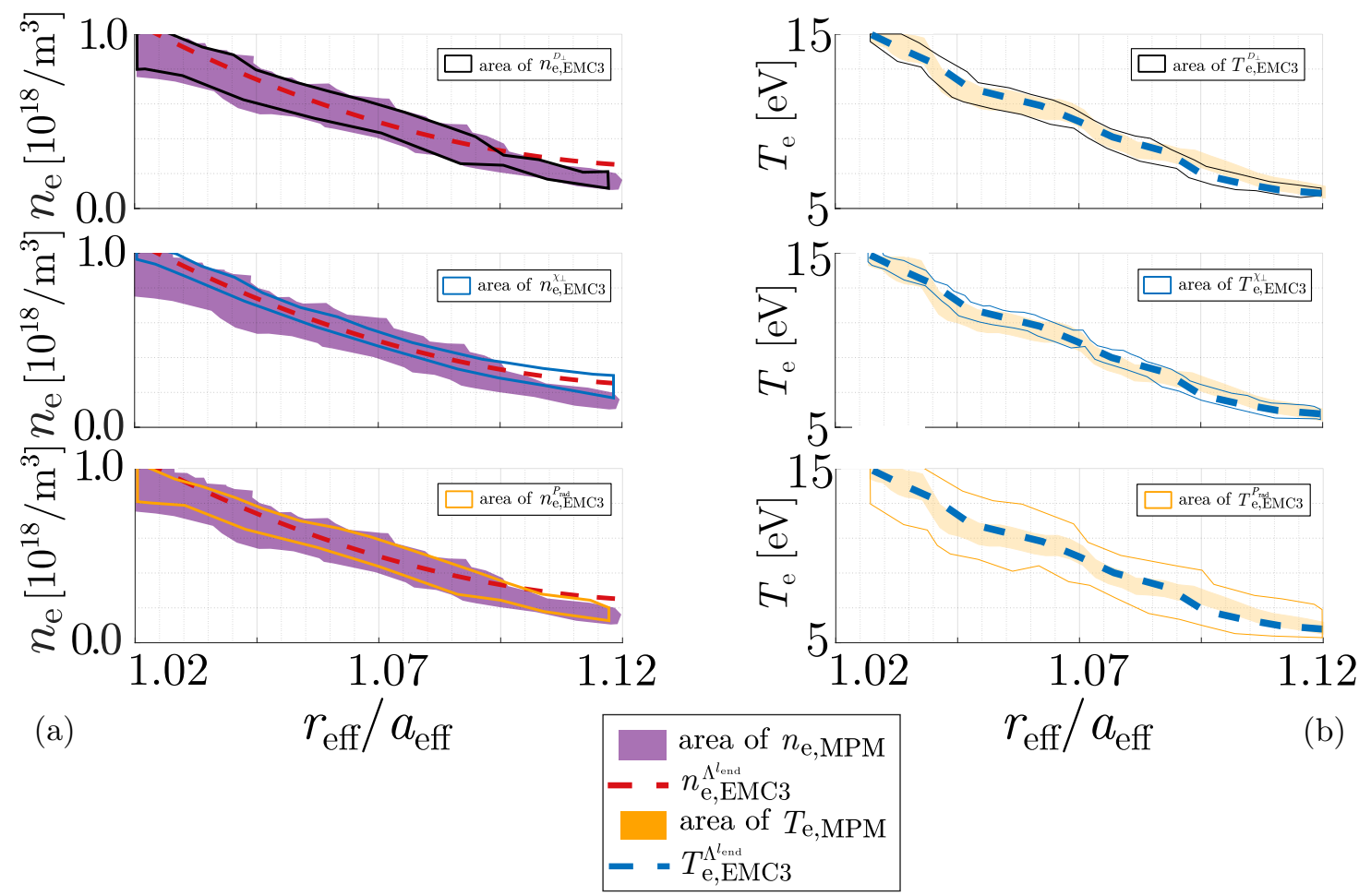

Figure 7. Overview of the influence of variation over $D_{\perp}, \chi_{\perp}$, and $P_{\mathrm{rad}}^{\mathrm{SOL}}$ on $n_{\mathrm{e}, \mathrm{EMC} 3}$ and $T_{\mathrm{e}, \mathrm{EMC} 3}$, plotted in translucent areas that entail the simulation results for $n_{\mathrm{e}, \mathrm{EMC} 3}^{D_{\perp}}$, $n_{\mathrm{e}, \mathrm{EMC} 3}^{\chi_{\perp}}, n_{\mathrm{e}, \mathrm{EMC} 3}^{\mathrm{P}} T_{\mathrm{e}, \mathrm{EMC} 3}^{\mathrm{SOL}}, T_{\mathrm{e}, \mathrm{EMC} 3}^{\chi_{\perp}}$, and $T_{\mathrm{e}, \mathrm{EMC} 3}^{\mathrm{P}_{\mathrm{rad}}^{\mathrm{SOL}}}$. These are plotted in comparison with the magenta and yellow shaded area of $n_{\mathrm{e}, \mathrm{MPM}}$ (in (a)) and $T_{\mathrm{e}, \mathrm{MPM}}\left(\right.$ in $(\mathrm{b})$ ); $n_{\mathrm{e}, \mathrm{EMC} 3}^{\Lambda_{\text {end }}}$ and $T_{\mathrm{e}, \mathrm{EMC} 3}^{\Lambda_{\text {end }}}$ are a guide to the eye.

equal to 1 is proven to be plausible. Reinterpreting the Langmuir probe measurements presented in this work appears to be irrelevant, at first glance. However, these Langmuir probe measurements were used as reference points to consistently assess missing $Z_{\text {eff }}$ and $m_{\text {eff }}$ profiles. The assessed $Z_{\text {eff }}$ profiles lie reasonably close (a maximum difference of $30 \%)$ to the made assumptions $\left(Z_{\text {eff }}=1.0\right)$. We inferred that the considered discharges reflect discharges with low levels of contamination with impurities, which is shown in figure 8. A relatively low radiated power was measured for the shown plasma scenario $\Lambda$ (see figure 1)). This measure is directly linked to the amount of impurities that soil the plasma scenario, and provides a plausible explanation for the minor impact of the reinterpretation process.

\section{Conclusion}

In this work, interpretative EMC3-EIRENE simulations were used to assess plasma parameters that were not directly accessible by any diagnostic available on W7-X in the first operational phase. Inferred spatial $Z_{\text {eff }}$ and $m_{\text {eff }}$ profiles were identified as 
further sensitive input information for the data processing of several W7-X diagnostics, especially Langmuir probes, because these diagnostics rely on the knowledge of radial $Z_{\text {eff }}$ profiles at their measurement position. Iterative reprocessing of the Langmuir probe data was performed for a comparison with EMC3-EIRENE simulations. This finally led to a fully $3 \mathrm{D}$ resolved edge-plasma simulation that lay in overall agreement with the available MPM and limiter Langmuir probe measurements (not discussed in this work), including $Z_{\text {eff }}$ being consistent with the measured radiative power losses. We concluded that more generally in 3D magnetic configurations, edge-plasma diagnostics - which rely on assumptions over spatial plasma parameter distributions such as $Z_{\text {eff }}$ - can profit from this complementary numerical diagnostic. Further model refinements, in particular in the trace impurity ion fluid model and comparison of simulations to further W7-X diagnostics, are steps that must be taken in the future, particularly when oxygen with its (hitherto neglected) accidental charge exchange resonance - remains a relevant plasma impurity in W7-X discharges. Non-uniform cross field must be studied alongside the impurity transport in the near future. Furthermore, oxygen impurities will have a higher impact on $Z_{\text {eff }}$ and $m_{\text {eff }}$ profiles than carbon as a single impurity. The impact of the reinterpretation process will become more pronounced, and therefore, discharges of operational phase 1.2 in a divertor configuration will be evaluated regarding this impact. However, the next step is to conduct the same refinement process of Langmuir probe data-sets with a numerically assessed saturation current rather than each charge state density. 


\section{Supplement}
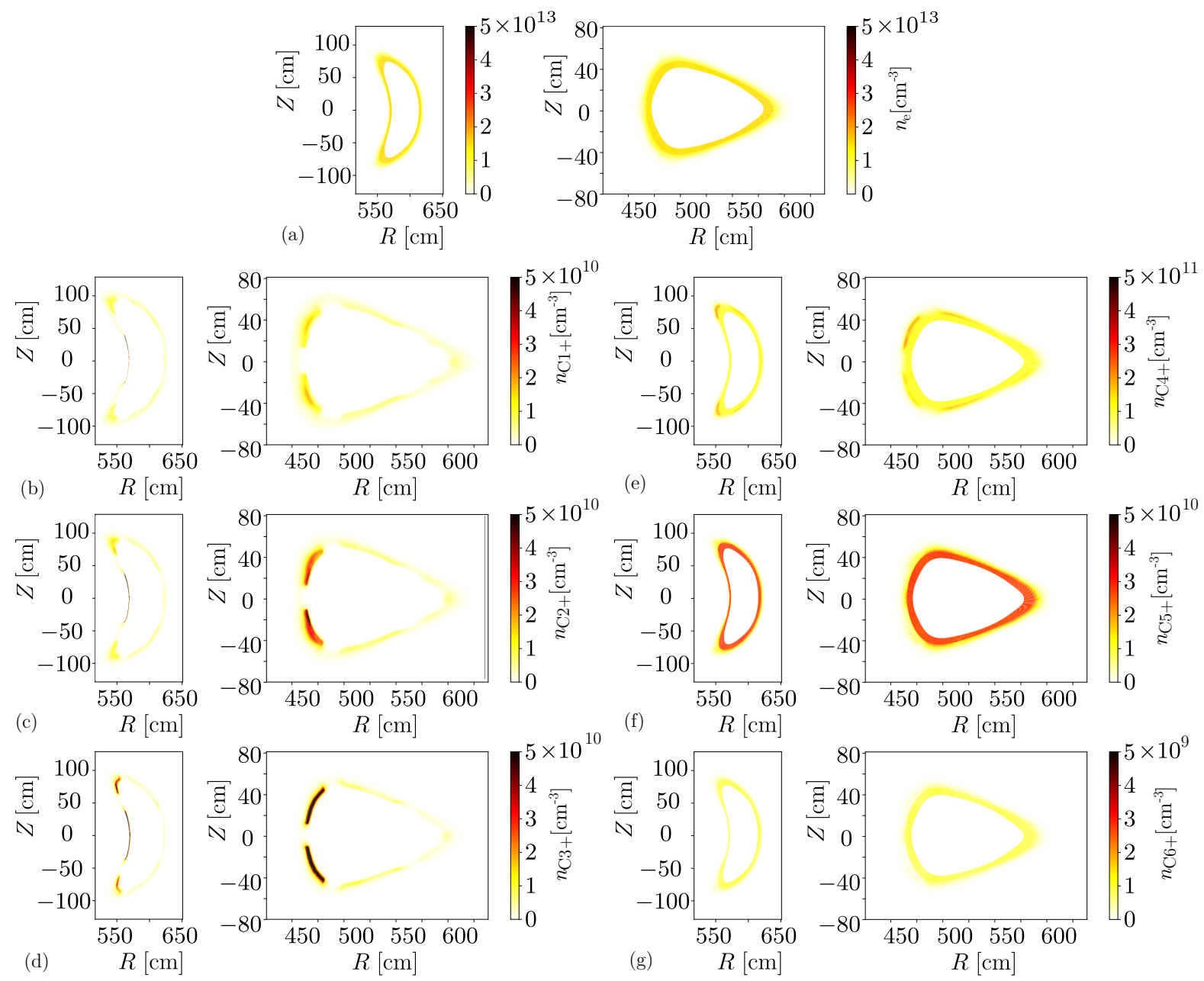

Figure 8. Simulated electron (a) and carbon charge state densities $\left(n_{\mathrm{C}^{1+}}\right.$ to $n_{\mathrm{C}^{6+}}$ in (b) to (g)) plotted in the cross section of $\varphi=0^{\circ}$ and $36^{\circ}$. The color code gives the magnitude of the density.

\section{Acknowledgments}

We are thankful for the valuable discussions with M. Rack, Dirk Reiser, Felix Reimold and Glen Wurden. The authors gratefully acknowledge the computing time granted by the JARA-HPC Vergabegremium and VSR commission on the supercomputer JURECA at Forschungszentrum Jülich. This work has been carried out within the framework of the EUROfusion Consortium and has received funding from the Euratom research and training programme 2014-2018 under grant agreement No. 633053. The views and opinions expressed here in do not necessarily reflect those of the European Commission. 


\section{References}

[1] Klinger T et al 2017 Plasma Phys. and Controll. Fusion 59014018

[2] Beidler C et al 1990 Fusion Technol. 17148

[3] Iiyoshi A et al 1990 Fusion Technol. 17169

[4] Pedersen T S et al 2015 Nucl. Fusion 55126001

[5] Wolf R C et al 2017 Nucl. Fusion 57102020

[6] Drews P et al 2017 Nucl. Fusion 57126020

[7] Nicolai D et al 2017 Fusion Eng. and Des. 123960

[8] Satheeswaran G. et al 2017 Fusion Eng. and Des. 123699

[9] Morita S et al 2001 Phys. Scr. 200148

[10] Dai S et al Nucl. Fusion 56066005

[11] Meister H et al 2003 Rev. Sci. Instrum., 744625

[12] Kobayashi M et al 2018 Contribut. Plasma Phys. 54383

[13] Drews P 2019 The role of magnetic topology in the edge plasma performance on $W 7-X \mathrm{PhD}$ thesis, Heinrich Heine University Düsseldorf

[14] Cosfeld J et al 2019 Nucl. Mater. Energy 18307

[15] Stangeby P C 2000 The Plasma Boundary of Magnetic Fusion Devices (Boca Raton: CRC Press)

[16] Tokar M Z 1994 Contribut. Plasma Phys 34139 